Haendel, A., Anderson, J. G., Pilz, M., Cotton, F. (2020): A Frequency-Dependent Model for the Shape of the Fourier Amplitude Spectrum of Acceleration at High Frequencies. - Bulletin of the Seismological Society of America, 110, 6, 2743-2754.

https://doi.org/10.1785/0120200118 


\title{
A frequency-dependent model for the shape of the Fourier amplitude spectrum of acceleration at high frequencies
}

\author{
Annabel Haendel, John G. Anderson, Marco Pilz and \\ Fabrice Cotton \\ in Bulletin of the Seismological Society of America
}

15 September 2020

\begin{abstract}
The high-frequency decay term of the acceleration spectrum $\kappa$ is a commonly used parameter in engineering seismology. In recent years, it has been recognized that the assumption of a linearly decaying spectrum in log-linear space is not always valid, as the value of $\kappa$ depends on the analyzed frequency band. We present an alternative model for the spectral fall-off in which the frequency dependence is explicitly taken into account. This is motivated by observations that the quality factor $\mathrm{Q}$ has a power-law dependence on frequency at high frequencies. The new model describes the spectral decay with the help of two variables, opposite to the single parameter $\kappa$. The approach is applied to borehole data of the EUROSEISTEST site in Greece. The misfit between modeled and observed spectra is reduced with the new approach compared to the classical kappa model. The new estimates compare well to $\kappa$ estimates if the same frequency interval is considered but allows additionally to capture the frequency-dependence of the spectral shape.
\end{abstract}

\section{Introduction}

In seismology, it is well known that the recorded acceleration amplitude spectra of earthquakes deviate from the idealized source model of Brune (1970) 
at frequencies higher than the source corner frequency. Instead of a flat spectrum, Fourier amplitudes of acceleration rapidly decrease above a certain frequency. This is easily explained by attenuation, for example, due to absorption processes and scattering along the propagation path and below the site. Understanding the spectral fall-off is of utmost importance as it governs the observed ground motions at a target site at high frequencies.

Hanks (1982) first termed $f_{\max }$ as the frequency above which the spectrum decays rapidly. Soon after, Anderson and Hough (1984) introduced the empirical parameter $\kappa$ to model the amplitude reduction.

They observed that the decay of the acceleration spectrum a(f) of S-waves follows an exponential function of the form:

$$
a(f)=a_{0} \cdot \exp [-\pi f \kappa] \quad\left(f>f_{e}\right)
$$

In Eq. 1, $f_{e}$ is sufficiently greater than the corner frequency of the earthquake, so that the source spectrum is assumed to reach its high-frequency asymptote. When taking the logarithm of Eq. 1 and plotting against linear frequency, the decay follows a linear trend. $\kappa$ can thus be simply computed from the slope above $f_{e}$ :

$$
\frac{d \ln (a(f))}{d f}=-\pi \kappa
$$

Anderson and Hough (1984) also noticed that the observed values of $\kappa$ at a site show a roughly linear increase with distance $(r)$. They proposed that $\kappa$ could be split into two components:

$$
\kappa=\kappa_{r} \cdot r+\kappa_{0}
$$

Although $\kappa$ was introduced entirely as an observational parameter, and the physics behind it is not completely understood (Ktenidou et al., 2014), early studies associated it with attenuation. From this perspective, the slope $\kappa_{r}$ is a regional effect that corresponds to the attenuation of S-waves traveling almost horizontally through the crust. The intercept at zero distance is $\kappa_{0}$. This term is usually considered to be mainly caused by the site-specific S-wave damping in the shallow crust (e.g. Hanks, 1982; Anderson and Hough, 1984; Ktenidou et al., 2013). Alternative models attribute $\kappa_{0}$ to source properties or attenuation close to the source (e.g Papageorgiou and Aki, 1983; Beresnev, 2019), or to a combination of both (e.g Purvance and Anderson, 2003; Van Houtte et al., 2011). 
Since its introduction, $\kappa$ has been widely used in the seismological and engineering communities for many regions in the world. It is, for example, applied in the simulation of stochastic ground motion (e.g Boore, 2003) or ground-motion model adjustment from a host to a target region (e.g. Al Atik et al., 2014; Campbell, 2003). Moreover, it is becoming more and more common to perform site-specific seismic hazard analysis, especially for critical facilities, for which the adjustment of $\kappa$ to hard rock conditions is necessary (e.g. Bommer et al., 2013; Biro and Renault, 2012).

A critical question is the relation between $\kappa$ and the seismic quality factor Q. Q is a physical property of the medium that is defined as the fractional loss of energy per cycle of oscillation (Knopoff, 1964). To be specific, for a monochromatic plane wave traveling in the $\mathrm{x}$-direction with velocity $\mathrm{c}$,

$$
a(x)=a_{0} \exp \left[\frac{-\pi r x}{c Q}\right]
$$

(Aki and Richards, 2002). Comparing Eq. 4 with Eq. 1 suggests, for a constant $\mathrm{Q}$, an equivalence of $\kappa$ and $x / c Q$ above a certain frequency threshold $f_{e}$

$$
\kappa=\frac{x}{c Q} \quad\left(f>f_{e}\right)
$$

Anderson and Hough (1984) have pointed out that three assumptions need to be met for Eq.5 to hold. The first is that the source acceleration spectrum is flat above the corner frequency of the earthquake, i.e. the spectrum follows an $\omega^{2}$ shape after smoothing over the fine structure. Second, the site response at high frequencies should also be approximately flat, so if the site has a predominant resonant frequency, $f_{e}$ should be above it. Third, Q needs to be independent of frequency. However, in contradiction to this third condition, in general, $\mathrm{Q}$ is a function of frequency, i.e. $\mathrm{Q}(\mathrm{f})$. This has been shown by many studies in the seismological literature (e.g. Aki, 1980a; Hellweg et al., 1995; Shi et al., 1996; Havskov et al., 2016; Fukushima et al., 2016). Q is, for example, observed to be depth-dependent on all spatial scales (e.g. Knopoff, 1964; Assimaki et al., 2008; Pasyanos, 2013; Pitilakis et al., 1999). Additionally, the inverse of the total (apparent) attenuation is a sum of two mechanisms - intrinsic absorption $\left(Q_{i}^{-1}\right)$ that is linked to the conversion of wave energy into heat, and scattering attenuation $\left(Q_{s c}^{-1}\right)$ that is caused by the scattering of the seismic waves at heterogeneities along the propagation path. While $Q_{s c}$ is generally assumed to be frequency-dependent (Aki, 


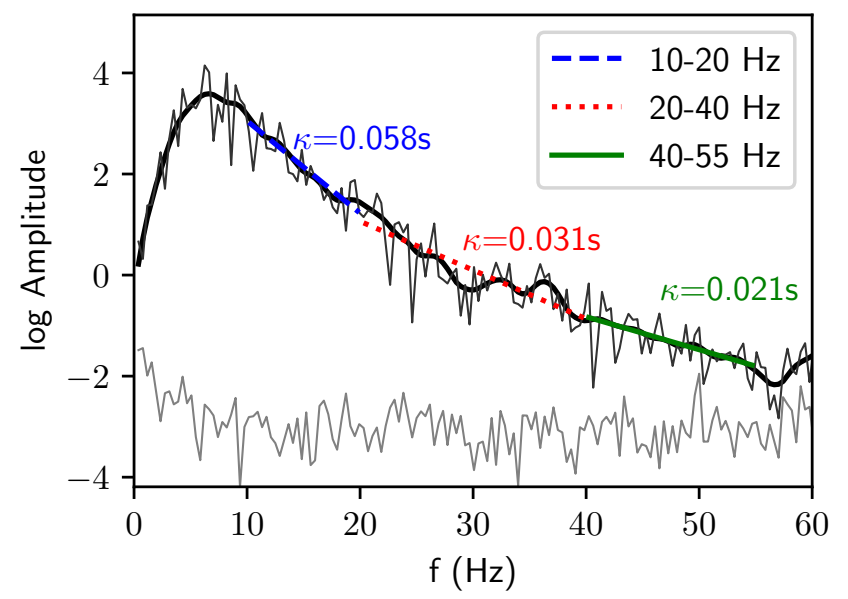

Figure 1: Example S-wave spectrum (dark grey), its smoothed version (bold black line) and the noise level (light gray) for a M3.7 earthquake recorded in $16 \mathrm{~km}$ distance at sensor '06' of the TST borehole in EUROSEISTEST Greece (compare to Figs 2 and 3). Show is the linear $\kappa$ fit for different choices of the frequency band.

1980b), $Q_{i}$ is often considered to be independent of frequency. Yet, several studies report a frequency-dependent $Q_{i}$ (e.g. Carcolé and Sato, 2010; Galluzzo et al., 2015; Eulenfeld and Wegler, 2016). For the purpose of this study, it is insignificant whether the frequency-dependence is caused by intrinsic or scattering attenuation or a combination of both. The common observation simply is that the apparent Q depends on frequency. Eq. 5 implies, then, that the slope of the spectrum, $d \ln a / d f$, would depend on $\mathrm{f}$ if the first two conditions (flat source and site spectrum at high frequencies) hold.

The data used by Anderson and Hough (1984) was not sufficient to detect a frequency band dependence of the spectral slope. Many recent studies (e.g. Edwards et al., 2015; Ktenidou et al., 2015; Mayor et al., 2018), however, suggest that the slope of the acceleration spectrum in log-linear space (Eq. 2) is not constant but rather curved so that the estimated $\kappa$ does depend on the chosen frequency band of analysis (compare to Fig. 1). Throughout this paper, use of the term "nonlinear" refers to this observation that the shape of the spectrum is curved in contrast to the linear kappa model shape. Nonlinearity in the stress-strain relation of the rock or soil is not implied.

We propose an alternative to the original kappa model of Anderson and 
Hough (1984) in which we incorporate a power-law dependence of Q. In doing so, the spectral decay at high frequencies becomes frequency-dependent. The nonlinear amplitude reduction is captured by two parameters $(\zeta$ and $\eta)$. We name the new model the zeta $(\zeta)$ model to distinguish it from the classical kappa model. In accordance with $\kappa$, it is possible to recognize both distance and site contributions to $\zeta$, corresponding to the terms in Eq. 3. The frequency dependence is introduced when $\eta>0$. In this publication, we test whether the updated model is consistent with the nonlinear spectral decay of real observations. The approach is applied to earthquakes recorded in a borehole array of accelerometers in EUROSEISTEST, Greece, and compared to $\kappa$ estimates of the original Anderson and Hough (1984) model.

\section{Methodology}

The general form of an S-wave acceleration spectrum measured at a distance $\mathrm{r}$ from a source can be described as:

$$
a(f)=E(f) P(f) S(f)
$$

where $\mathrm{E}(\mathrm{f}), \mathrm{P}(\mathrm{f})$ and $\mathrm{S}(\mathrm{f})$ are the frequency-dependent contributions to the spectrum from the earthquake source, path, and site, respectively. The path term is usually of the following form:

$$
P(f)=\frac{1}{r^{n}} \exp \left[-\frac{\pi f r}{Q(f) \beta}\right]
$$

In Eq. $7,1 / r^{n}$ is due to geometrical spreading with distance $\mathrm{r}$ where $n=1$ for body waves in a homogeneous medium. The exponential function describes the attenuation along the path with $\beta$ being the shear-wave velocity and $Q(f)$ the quality factor of shear waves.

Assuming that $Q(f)$ is a constant $Q_{c}$ independent of frequency, the exponent may be rewritten with the help of the attenuation parameter $t^{*}$, that is defined as the integrated value of $\mathrm{Q}^{-1}$ along the ray path:

$$
t^{*}=\int_{\text {path }} \frac{d r}{Q_{c}(r) \beta(r)} \simeq \frac{r}{Q_{c} \beta}
$$

Assuming additionally that the source $E(f)$ and the site $S(f)$ spectra are flat at high frequencies above the threshold $f_{e}$, we can claim that $t^{*}$ in 
Eq. 8 is equivalent to $\kappa$. Merging the source and site components with the geometrical spreading term into $a_{0}$, we can re-write Eq. 6 into the well known equation of Anderson and Hough (1984):

$$
a(f)=a_{0} \cdot \exp \left[-\frac{\pi f r}{Q_{c} \beta}\right]=a_{0} \cdot \exp [-\pi f \kappa] \quad\left(f>f_{e}\right)
$$

We will call this the "kappa model" for the high-frequency shape that is only valid above $f_{e}$.

Now let us preserve the aforementioned assumption of a flat source and site spectrum at high frequencies, but incorporate the observations of a frequency-dependent Q at high frequencies (e.g. Aki, 1980a,b; Aki and Chouet, 1975; Singh and Herrmann, 1983; Sato et al., 2012)

$$
Q(f)=Q_{0}\left(\frac{f}{f_{0}}\right)^{\eta}
$$

$f_{0}$ is a reference frequency. $Q_{0}$ is the value of $\mathrm{Q}$ at $f_{0}$ and the exponent $\eta$ is typically in the range of $0.5-1$ (Sato et al., 2012). The Q in Eq. 10 is an apparent $\mathrm{Q}$ that describes the overall attenuation due to absorption and scattering. Inserting Eq. 10 into Eq. 9, the result is

$$
a(f)=a_{0} \cdot \exp \left[\frac{-\pi r f}{Q_{0}\left(\frac{f}{f_{0}}\right)^{\eta} \beta}\right] \quad\left(f>f_{e}\right)
$$

Combining the frequency terms in the numerator and the denominator will lead to

$$
a(f)=a_{0} \cdot \exp \left[-\pi \frac{r f_{0}}{Q_{0} \beta}\left(\frac{f}{f_{0}}\right)^{1-\eta}\right] \quad\left(f>f_{e}\right)
$$

Setting

$$
\zeta_{f_{0}}=\frac{r f_{0}}{Q_{0} \beta}
$$

we obtain a frequency-dependent version of Eq. 9

$$
a(f)=a_{0} \cdot \exp \left[-\pi \zeta_{f_{0}}\left(\frac{f}{f_{0}}\right)^{1-\eta}\right] \quad\left(f>f_{e}\right)
$$


Instead of a frequency-independent term $\kappa$ in Eq. 9 with the unit seconds, Eq. 14 describes the frequency-dependent spectral decay at high frequencies with the help of the two variables $\zeta_{f_{0}}$ and $\eta$. Note that $\zeta_{f_{0}}$ is dimensionless in contrast to $\kappa$ with dimensions of time. $\zeta_{f_{0}}$ is dependent on the reference frequency $f_{0}$ so that e.g. $\zeta_{5}$ for $f_{0}=5 \mathrm{~Hz}$. Because $f_{0}=1 \mathrm{~Hz}$ is the most common reference frequency, we use the simple notation $\zeta$ when $f_{0}=1 \mathrm{~Hz}$. We will refer to Eq. 14 as the "zeta model" for the high frequency shape of the acceleration spectrum.

Taking the natural log of Eq. 14, and using the assumption that the spectrum would be flat except for the effect of attenuation, one finds

$$
\ln a(f)=\ln a_{0}-\pi \zeta_{f_{0}}\left(\frac{f}{f_{0}}\right)^{1-\eta} \quad\left(f>f_{e}\right)
$$

In the following, $\eta$ can be fixed in trial runs. For any selected value of $\eta$, let us suppose that optimized parameters to fit the high-frequency acceleration spectrum $d_{1}(f)=\ln a(f)$ yield

$$
\hat{d}_{1}(f \mid \eta)=c_{1}(\eta)+c_{2}(\eta)\left(\frac{f}{f_{0}}\right)^{1-\eta}
$$

where $c_{1}$ and $c_{2}$ are found by least squares. We can define the quality of fit to the high-frequency part of the spectrum by the standard deviation,

$$
\sigma_{1}^{2}(\eta)=\int\left\{\left[\hat{d}_{1}(f \mid \eta)-d_{1}(f)\right]^{2}\right\} d f
$$

For each seismogram, then we could choose the value of $\eta$ that minimizes $\sigma_{1}(\eta)$. However, using observed data we find that the minimum is shallow and thus $\zeta_{f_{0}}$ and $\eta$ are not well constrained by an individual seismogram. We therefore concluded that it is necessary to minimize the variance considering multiple seismograms recorded at a single station:

$$
\sigma_{K}^{2}(\eta)=\frac{1}{N_{k}} \sum_{k=1}^{N_{k}} \sigma_{k}^{2}(\eta)
$$

In this case, $N_{k}$ is the number of seismograms recorded at station $\mathrm{K}$. We then consider the value of $\eta$ that minimizes $\sigma_{K}(\eta)$ to be our best estimate. The solutions for $\ln a_{0}$ and $\zeta_{f_{0}}$ are derived from $c_{1}(\eta)$ and $c_{2}(\eta)$ that are obtained for the best estimate of $\eta$. 
Having estimates for $\eta$ and $\zeta_{f_{0}}$, we can compute the slope of spectral decay. In the traditional kappa model of Anderson and Hough (1984), the slope of the linear fit is equal to $-\pi \kappa$. For the frequency-dependent model in Eq. 15 the slope can be calculated as

$$
-\frac{1}{\pi} \frac{d \ln a(f)}{d f}=\frac{\zeta_{f_{0}}}{f_{0}}(1-\eta)\left(\frac{f}{f_{0}}\right)^{-\eta}=\kappa_{\eta, \zeta}(f)
$$

which can be thought of as a frequency-dependent $\kappa_{\eta, \zeta}(f)$. The values of $\kappa_{\eta, \zeta}(f)$ are equivalent for different choices of $f_{0}$. We will show subsequently that at short distances or where $\mathrm{Q}$ is high (small attenuation), there is a strong trade-off between $\eta$ and $\zeta_{f_{0}}$. In these cases, $\kappa_{\eta, \zeta}(f)$ is still well resolved. This would be expected because $\eta$ and $\zeta_{f_{0}}$ are parameters for a curve that fits the spectral data where $\kappa$ could be measured directly instead.

Following the classical kappa model, $\zeta_{f_{0}}$ can be plotted against epicentral distance $\mathrm{r}$ to find the value $\zeta_{f_{0}, 0}$ at $r=0$. Inserting $\zeta_{f 0,0}$ into Eq. 19 and solving the equation for different frequencies $\mathrm{f}$, the frequency-dependent equivalent of $\kappa_{0}$ is obtained:

$$
\kappa_{0}(f)=\frac{\zeta_{f_{0}, 0}}{f_{0}}(1-\eta)\left(\frac{f}{f_{0}}\right)^{-\eta}
$$

The unit of $\kappa_{0}(f)$ is also seconds. Similar to Eq. 19, Eq. 20 results in the same $\kappa_{0}(f)$ values for different choices of $f_{0}$.

\section{Data}

The proposed method is applied to data of the well-studied European experimental site EUROSEISTEST in Northern Greece (Pitilakis et al., 2013). The test site is situated in the tectonically active Mygdonia sedimentary basin that lies approximately $30 \mathrm{~km}$ to the northeast of the city of Thessaloniki (Fig. 2). A permanent seismic network of 14 surface and 6 downhole accelerographs is installed throughout the valley (Pitilakis et al., 2013) with the first sensors starting to operate in 1993.

We use data of the TST borehole that is located in the center of the Mygdonia valley. 6 accelerographs are deployed at 0, 18, 40, 73, 136 and $196 \mathrm{~m}$ depth. The shear-wave velocity profile of the selected site is shown in Fig. 3. The 5 uppermost sensors are located within the sedimentary fill of 

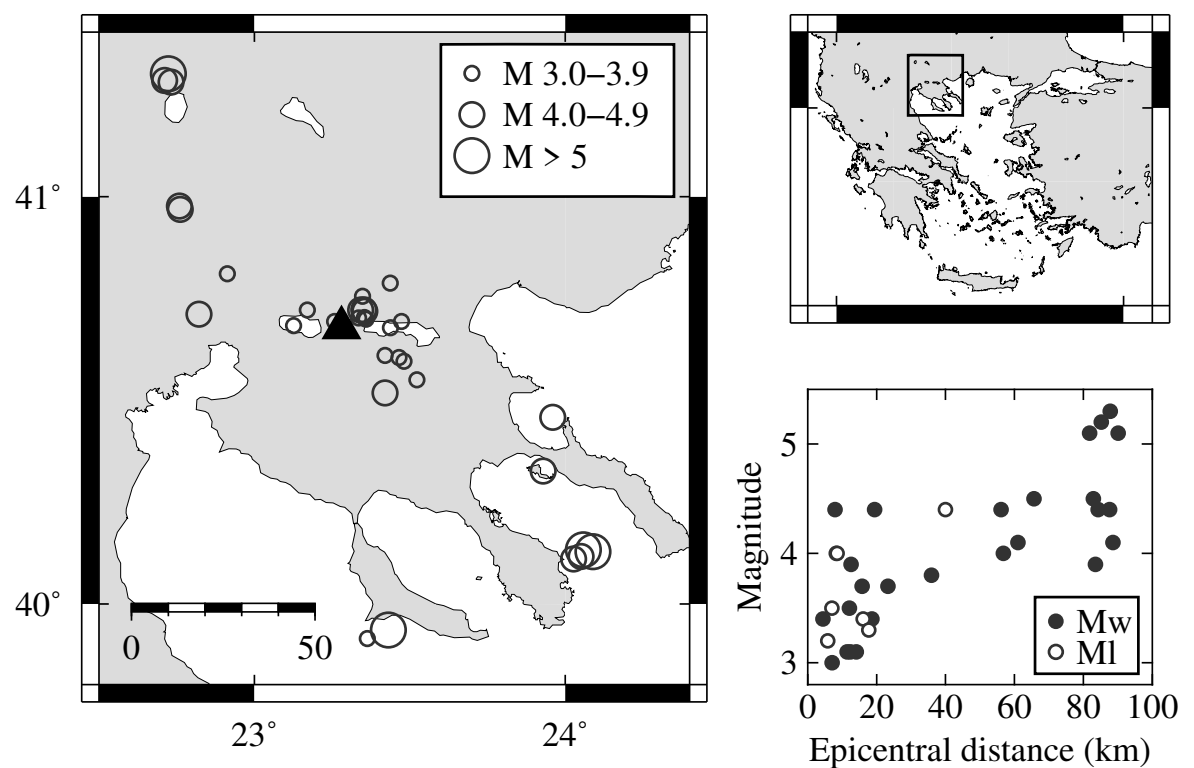

Figure 2: Left: Epicenters of the events used in this study (black circles) and location of the TST borehole (black triangle). Upper right: Location of the study area in Greece. Bottom right: Magnitude-distance distribution of the selected events. Moment magnitude Mw is used for most of the events. Local magnitude $\mathrm{Ml}$ is used if an estimate of $\mathrm{Mw}$ is not available. 


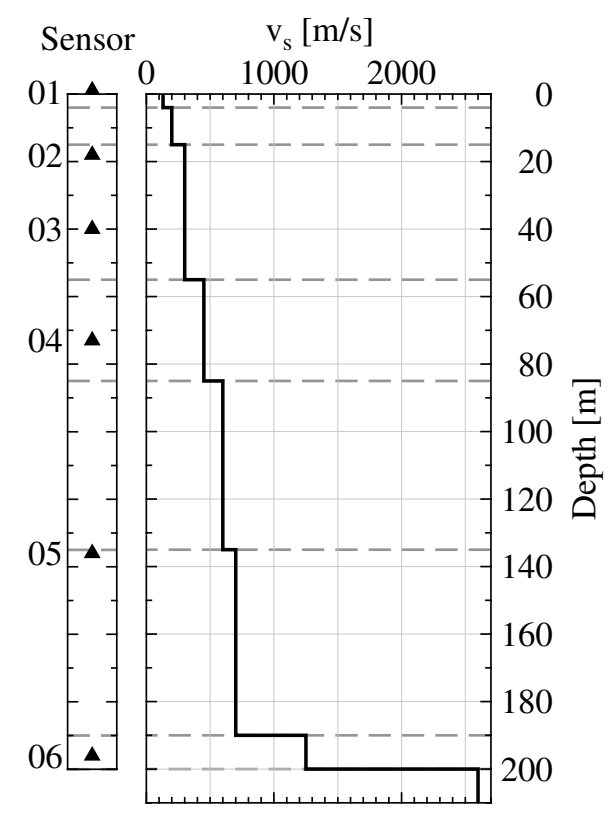

Figure 3: Sensors depth (black triangles) and the shear-wave velocity profile for the TST site (adapted from Jongmans et al., 1998). 
the basin. Only the deepest station is situated close to the bedrock level at $200 \mathrm{~m}$ depth.

A set of 33 earthquakes from the years 2003-2016 is extracted from the EUROSEISTEST database web portal (see Data and Resources Section). Distances are restricted to be smaller than $100 \mathrm{~km}$ where we can assume that the energy in the S-wave has primarily propagated only in the crust. Additionally, only events with a magnitude larger than 3 are selected to avoid the influence of the earthquake corner frequency on the $\kappa$-decay band. The magnitude-distance distribution of the selected earthquakes is shown in Fig. 2.

S-wave onsets are manually picked for the surface and the deepest station. Using the S-wave picks of the deepest sensor, S-wave arrivals for the intermediate stations can be approximated using the velocity profile given in Fig.3. The length of the S-wave window $\left(D_{S}\right)$ is set following the definition of Perron et al. (2018)

$$
D_{S}=\frac{1}{f_{c}}+\left(T_{S}-T_{P}\right)
$$

The second term in Eq. 21 takes into account the expansion of the signal along the propagation path approximated by the S-minus-P wave arrival time difference $\left(T_{S}-T_{P}\right)$. The first term shows the influence of the source through the inverse of the corner frequency $f_{c}$. $f_{c}$ is selected manually for each recording on the deepest sensor '06' by investigating both acceleration and displacement spectra.

Noise windows are taken between $[\mathrm{Tp}-3.5, \mathrm{Tp}-0.5] \mathrm{s}$. The extracted Swave and noise windows of the recordings are tapered with a $5 \%$ cosine function, padded with zeros and transformed to the Fourier domain. The amplitude spectra are smoothed with a moving average filter using a Hann window of $5 \mathrm{~Hz}$ length. The two horizontal spectra of an earthquake are combined into a single one by taking the root-mean-square (rms). We opted to average the horizontal components to reduce the influence of the azimuthal direction on the $\kappa, \eta$ and $\zeta$ measurements (Ji et al., 2020).

Finally, the frequency band for the estimation of the spectral decay needs to be defined. The lower frequency limit $\left(f_{e}\right)$ is set to be $f_{e}=f_{c}+5 \mathrm{~Hz}$. If $f_{e}<10 \mathrm{~Hz}, f_{e}=10 \mathrm{~Hz}$ is used instead to avoid the influence of down-going waves and of site resonance. According to Raptakis et al. (2005), the fundamental resonance frequency that is caused by the $200 \mathrm{~m}$ deep sedimentbedrock interface is observed at about $0.6 \mathrm{~Hz}$. Yet, the fundamental fre- 


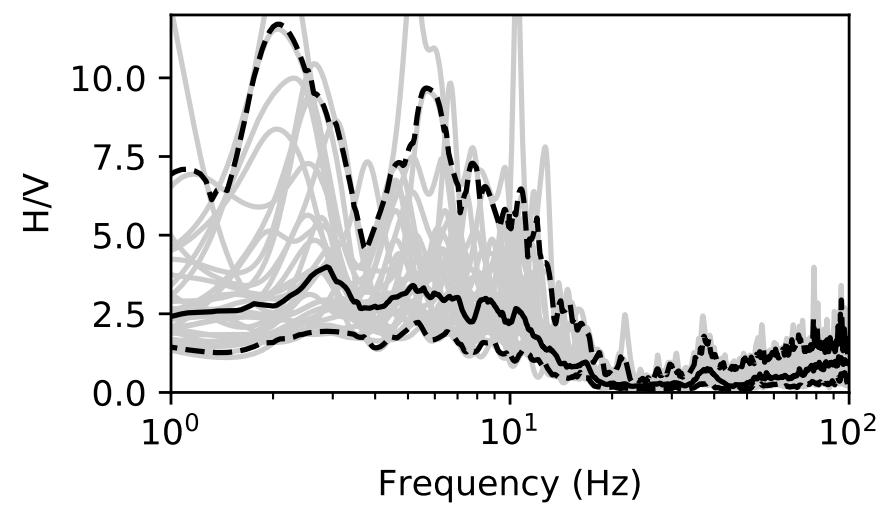

Figure 4: H/V spectral ratios of all earthquakes (gray) recorded at the TST surface station. The black solid and dashed lines show the median and the 3 and $97 \%$ percentiles of the curves.

quency is not necessarily the predominant frequency of the site (frequency where the amplification is largest). The horizontal-to-vertical (H/V) spectral ratio computed for the surface station at TST e.g. shows some amplification up to $10 \mathrm{~Hz}$ which could, for example, be caused by weathered layers. By setting $f_{e} \geq 10 \mathrm{~Hz}$, these amplifications are avoided. The upper-frequency limit $\left(f_{x}\right)$ is selected where the signal-to-noise ratio falls below 4 or it is set to $50 \mathrm{~Hz}$ if the signal is still strong enough. The choice of $f_{e}$ and $f_{x}$ is consistent with the recommendations of Ktenidou et al. (2013).

\section{Zeta model results}

We set $f_{0}=1 \mathrm{~Hz}$ in the subsequent analysis. This is in agreement with most studies that consider a frequency-dependent Q according to Eq. 10 (e.g. Aki, 1980a; Singh and Herrmann, 1983; Toksöz et al., 1988; Havskov et al., 2016; Wang and Shearer, 2019). As pointed out in the Methodology section, we use the notation $\zeta$ instead of $\zeta_{1}$ at $f_{0}=1 \mathrm{~Hz}$.

Fig. 5 shows the standard deviation of the misfit $\sigma(\eta)$ for different choices of $\eta$. The result is given exemplary for the deepest sensor '06'. The grey curves depict $\sigma(\eta)$ for the 33 individual recordings of the events that are introduced in Fig. 2. We note that the minima for individual records (gray dots) are rather shallow, indicating that a tradeoff of $\eta$ and $\zeta$ is allowed by the 


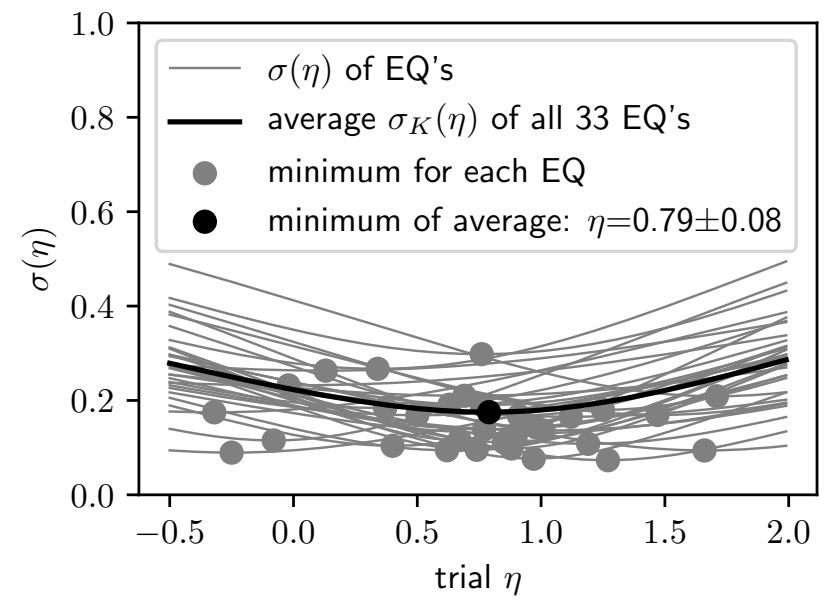

Figure 5: $\sigma(\eta)$ computed using Eq. 17 as a function of trial $\eta$ for station '06'. Grey lines show $\sigma(\eta)$ obtained for the recordings of the 33 events introduced in Fig. 2. The black line represents the average of all gray lines (Eq. 18). The minima of the curves are denoted by grey and black points.

data. The best estimate of $\eta$ thus varies for individual earthquakes between -0.4 and 1.75. Due to the large scatter of "best- $\eta$ " estimates between different earthquakes we also minimized the variance considering all events together. This is done by taking the average of all gray lines according to Eq. 18 which results in the black curve. The black curve minimizes for $\eta=0.79$. This value corresponds to the best estimate of $\eta$ for station '06'. We performed a jacknife resampling (Efron and Stein, 1981) to find the confidence interval of the estimated value of $\eta$. The standard error of the jacknife test gives a confidence interval of \pm 0.08 for station '06'.

A plot of $\zeta$ as a function of epicentral distance is given in Fig. 6 for stations '06' and '01'. We used a robust regression to avoid the bias due to outliers. The distance regression yields an intercept at zero distance $\left(\zeta_{0}\right)$. This value can be used in Eq. 20 to derive $\kappa_{0}(f)$. The slope of the regression line $(d \zeta / d r)$ is related to the regional $Q_{0}\left(\mathrm{Q}\right.$ at $f_{0}=1 \mathrm{~Hz}$ ) via the derivative of Eq. 13 and for $f_{0}=1 \mathrm{~Hz}$ :

$$
\frac{d \zeta}{d r}=\frac{1}{Q_{0} \beta},
$$

We have carried out this analysis for all stations of the TST borehole. 

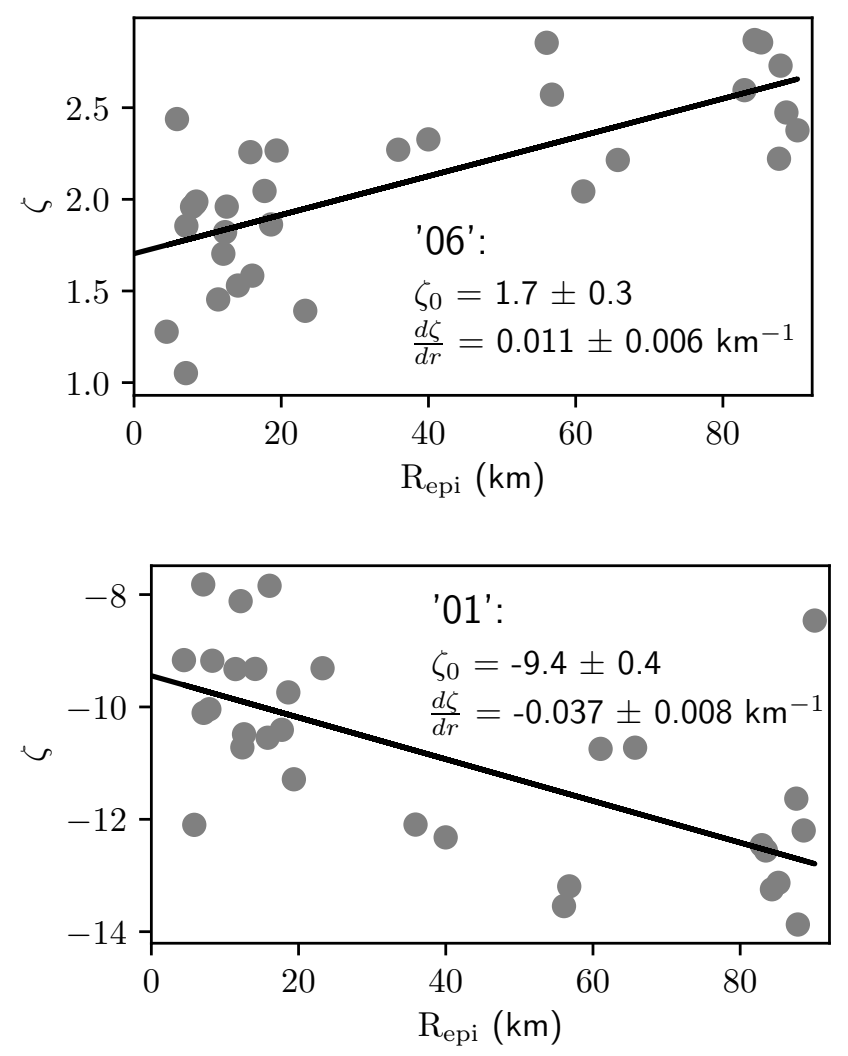

Figure 6: Regression of $\zeta$ with epicentral distance for station '06' (left) and station '01' (right). The intersect at zero distance gives the value of $\zeta_{0}$. 

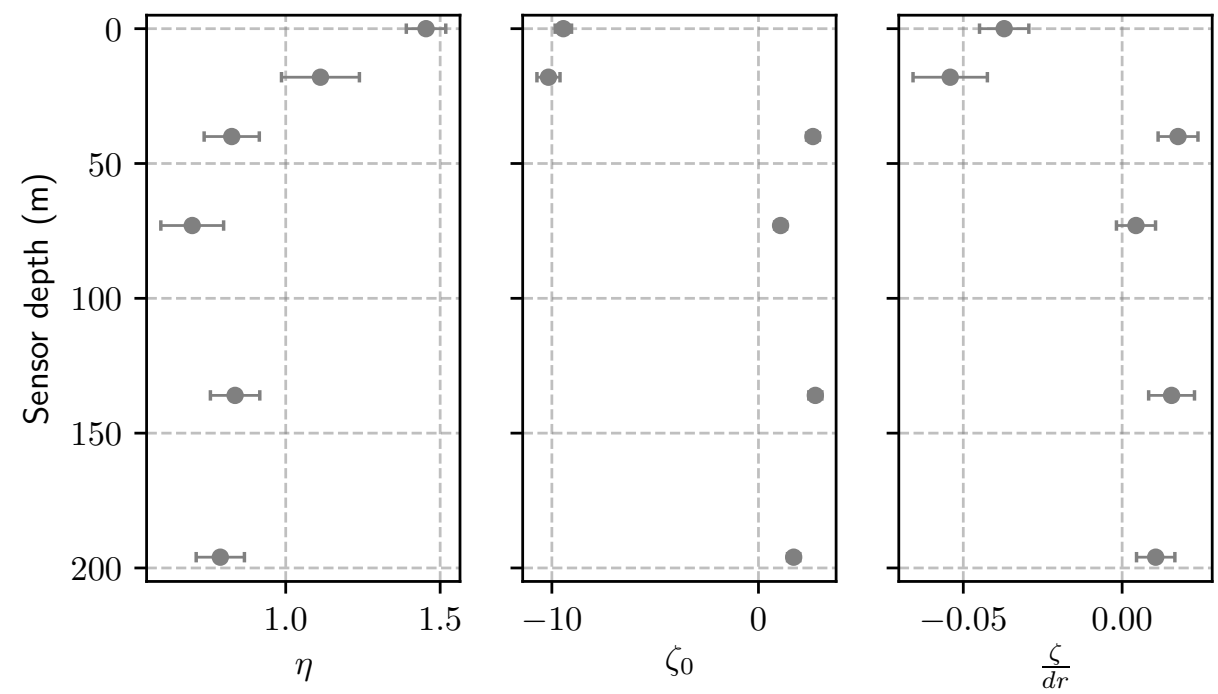

Figure 7: Results of the zeta model for all stations. Estimated $\eta$ (left), the zero-distance intercept $\zeta_{0}$ (middle) and the slope of the distance regression $\frac{d \zeta}{d r}$ (right) as a function of station depth. The confidence intervals of the estimates are shown as errorbars. 
Fig. 7 summarizes the inversion results and the corresponding errors for all stations. $\eta$ scatters around 0.8 at the four deepest sensors and has values of $\eta>1$ for the stations at the surface and in $18 \mathrm{~m}$ depth. As can be seen in Figs 6 and 7 , values of $\zeta$, the slope $d \zeta / d r$ and the intercept $\zeta_{0}$ are positive for sensor '03'-'06' and negative for sensors '01' and '02'. $\zeta_{0}$ varies between 1-3 for the deepest stations and is negative for stations '01' and '02'. The slope $d \zeta / d r$ leads to estimates of $0.005-0.02 \mathrm{~km}^{-1}$ at the four deepest sensors and to negative values at the two uppermost stations. This is due to $\eta>1$ for sensor '01' and '02'. A negative slope would indicate a negative regional Q, which contradicts the conservation of energy, the findings of the deeper stations in the TST borehole and of other attenuation studies in the area. We note that the negative $\zeta$ values for station '01' and '02' are compensated by the factor $(1-\eta)<0$ in the computation of $\kappa_{\eta, \zeta}(f)$ so that, finally, $\kappa_{\eta, \zeta}(f)>0$ (compare to Eq. 19).

The difference between $\zeta$ and $\eta$ estimates between sensors '01' and '02' and the deeper sensors is a graphic reminder that the site term $\mathrm{S}$ (f) (Eq.6) might have a major effect on the spectral shape. The $\mathrm{H} / \mathrm{V}$ spectral ratio of station '01' in Fig. 4 is not completely flat above $10 \mathrm{~Hz}$. Sensor '02', on the other hand, could be affected by the higher modes of downgoing waves which are still visible above $10 \mathrm{~Hz}$ (plot not shown here). Some of the observed differences might be an effect of the angle of incidence that varies as a function of depth of the station. Finally, the frequency dependence of $\mathrm{Q}$ in shallow and deeper geological structures may be entirely different. Revealing such effects could be regarded as an advantage of the zeta model, that will lead eventually to a deeper understanding of the effects of shallow geology on high frequency ground motions.

When studying vertical arrays, researchers usually constrain a common slope $(d \kappa / d r)$ for all sensors including the surface station (Ktenidou et al., 2013, 2015) or only from the sensors at depths (Van Houtte et al., 2011). This is based on the idea that the stations in a vertical array are close to each other and experience the same regional attenuation. For the zeta model, the situation is slightly more difficult. A common trend of $\zeta$ with epicentral distance is obtained only if $\eta$ is common for all stations. The inversion results shown in Fig. 7 suggest, that a common distance regression should only be performed for the four deepest sensors of the TST borehole. For test purposes, we derived a best-fitting $\eta$ from all spectra recorded at stations '03'-'06' which results in $\eta=0.79 \pm 0.05$. It was then possible to do a joined distance regression for these stations which gives a common regional slope of 


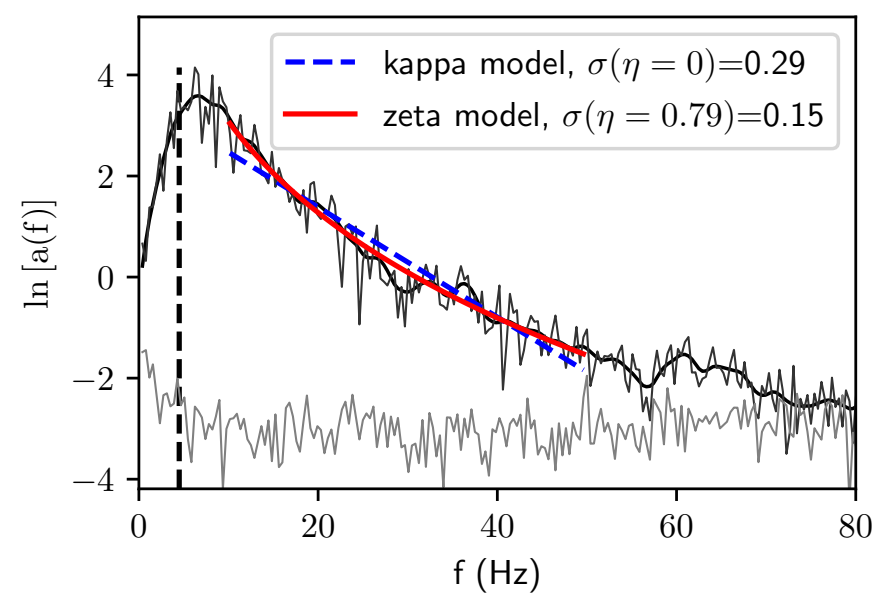

Figure 8: Example S-wave spectrum at station '06' (dark grey) of a M 3.7 earthquake in $16 \mathrm{~km}$ distance, the smoothed spectrum (black) and the noise level (light gray). The vertical dashed line corresponds to the source corner frequency. The blue, dashed line shows the fit using the kappa model. The red, solid line is the result of the zeta model. The standard deviation of the misfit $\sigma(\eta)$ of the two fits is given in the legend.

$d \zeta / d r=0.010 \pm 0.001 \mathrm{~km}^{-1}$ We do not show these results in a figure since they are very similar to the outcomes of station ' 06 ' presented in Figs 5 and 6.

\section{Comparison of the zeta and the kappa model}

Fig. 8 shows the resulting fit of the kappa and the zeta model to an example acceleration spectrum of one earthquake. For the zeta model, the best estimate $\eta=0.79$ for station '06' (Fig. 5) was used. For the kappa model, $\eta$ is equal to zero. The standard deviation of the misfit $\sigma(\eta)$ almost halves by the use of the zeta-model for the chosen earthquake. Considering the spectra of all earthquakes recorded at station ' 06 ', $\sigma(\eta)$ reduces on average from 0.27 to 0.2 for the zeta model compared to the kappa model (Fig. 9). The reduction is even larger for the other stations as is plotted in Fig. 9.

According to Eq. 19, the slope of the zeta model, evaluated for a frequency $f_{c e n}$, is proportional to $\kappa_{\eta, \zeta}\left(f_{c e n}\right) . \kappa_{\eta, \zeta}\left(f_{c e n}\right)$ might be expected to be equal 


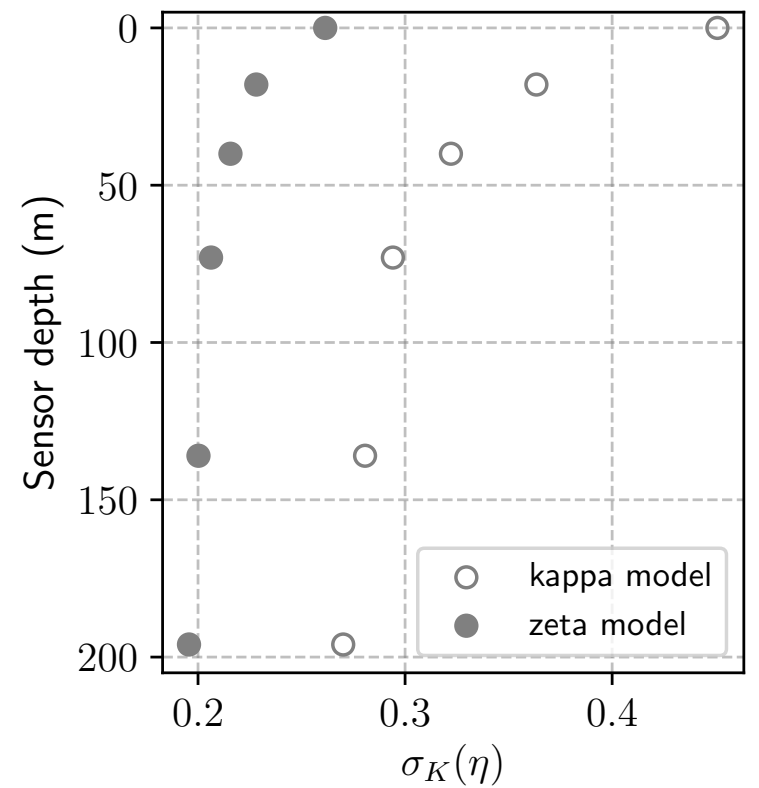

Figure 9: Average misfit for the kappa and the zeta model as a function of sensor depth computed after Eq. 18 and using the best estimates of $\eta$ as shown in Fig. 7. The average has been taken from the 33 events shown in Fig. 2 


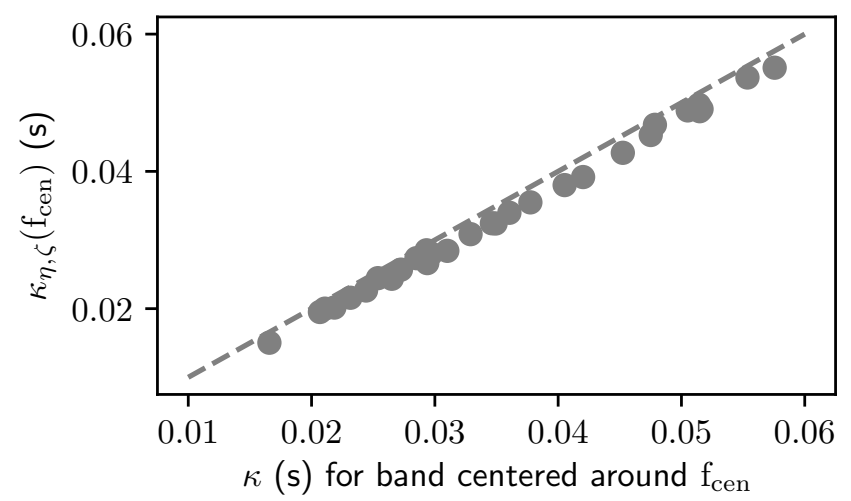

Figure 10: Comparison of the kappa model and the zeta model. According to Eq. $19, \kappa_{\eta, \zeta}$ evaluated for a frequency $f_{c e n}$ and $\kappa$, computed from the linear regression in a frequency band centered around $f_{c e n}$, should be similar. The center frequency is different for each spectrum according to the chosen values of $f_{e}$ and $f_{x}$. The dashed line corresponds to a line with slope $=1$. The result is shown for station ' 06 '.

to the value of $\kappa$, obtained from a kappa model fit for a frequency band centered around $f_{c e n}$. Fig. 10 confirms that the values of $\kappa$ and $\kappa_{\eta, \zeta}$ are in excellent agreement and follow an almost 1-to-1 correlation. This finding is encouraging and corroborates the idea that the kappa model of Anderson and Hough (1984) can be modified in the way proposed in this study to account for the curved shape of the spectral decay. The small differences between the $\kappa$ values derived with kappa and the zeta model that are visible in Fig. 10 are a consequence of the exponential form of the zeta model. If we consider the same frequency range and compute the slope of a linear function and of an exponential function at the center position $f_{c e n}$, the slope of the exponential function will always be slightly smaller than the slope of the linear model. The difference in the slope is, however, small (less than 0.3\%) as the observed acceleration spectra at Euroseistest are only slightly curved.

Using the kappa model, a careful selection of the frequency range will always be necessary to ensure that the spectral decay follows a linear trend. If the spectrum is curved due to frequency-dependent attenuation, the choice of a different frequency band will also lead to a different $\kappa$ estimate. While classical $\kappa$ studies have shown the difficulty of choosing a well-constrained frequency band, it is sufficient for the zeta model to select the frequency range 

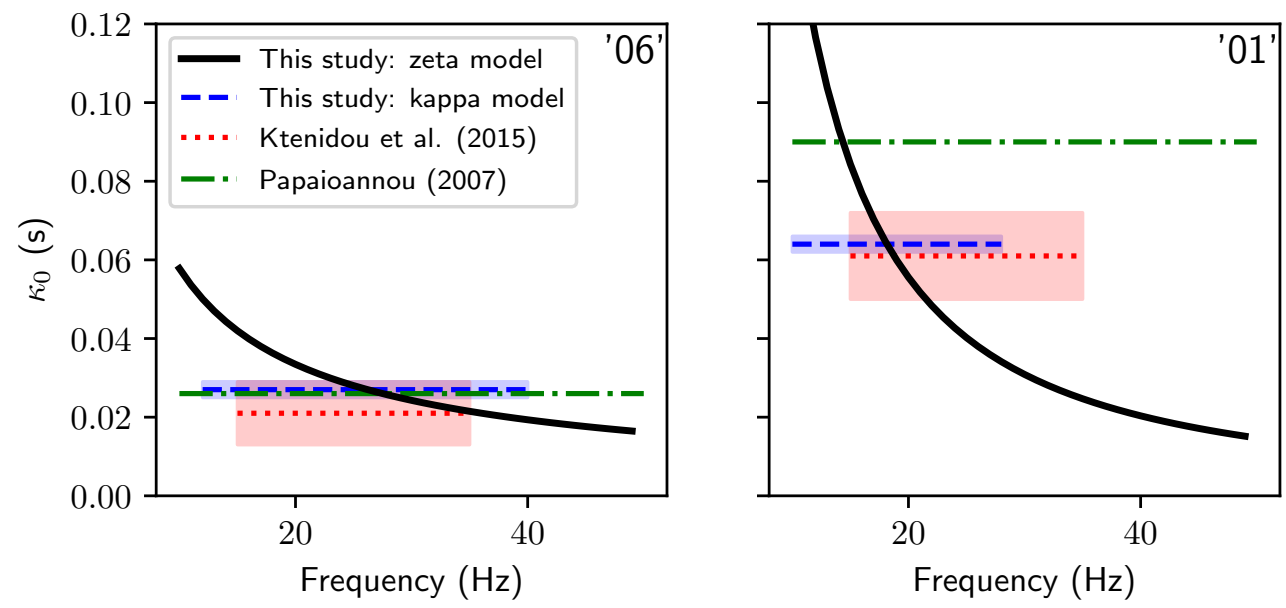

Figure 11: $\kappa_{0}$ estimates obtained from the zeta and the kappa model for station '06' (left) and station '01' (right). The black lines show the computed values of $\kappa_{0}$ as a function of frequency for the zeta model, based on Eq. 20 and the estimates of $\zeta$ and $\eta$ from Fig. 7 . The blue, dashed lines give the $\kappa_{0}$ values derived with the kappa model and the associated uncertainties (blue box). The mean frequency range used for the kappa model analysis (indicated by the $\mathrm{x}$-axis position and length of the blue lines) is $12-40 \mathrm{~Hz}$ for station '06' and $10-28 \mathrm{~Hz}$ for station '01'. The red and green lines are the results of Ktenidou et al. (2015) and Papaioannou (2007). The red box corresponds to the uncertainties reported by Ktenidou et al. (2015).

between a lower frequency, that is well above the source corner frequency $f_{c}$, and an upper frequency, where the signal-to-noise ratio falls below a chosen threshold. For each spectrum, only a single parameter set $(\eta, \zeta)$ is derived that can properly describe the nonlinear decay for all frequencies. The new model is thereby better suited for automation. Yet, similar to the kappa model, the chosen frequency bandwidth should be large enough to ensure that $\eta$ and $\zeta$ can be well constrained. Additionally, it needs to be verified that the curvature of the spectrum is only caused by frequency-dependent attenuation and not, for example, by site effects. Otherwise, these effects are mixed (Parolai and Bindi, 2004).

Fig. 11 shows $\kappa_{0}$ as a function of frequency computed with Eq. 20 for station '06' and station '01'. $\kappa_{0}$ decreases from $0.06 \mathrm{~s}$ to $0.02 \mathrm{~s}$ for frequencies between $10 \mathrm{~Hz}$ and $50 \mathrm{~Hz}$ for station '06'. For station '01', $\kappa_{0}$ is larger than 
$0.12 \mathrm{~s}$ at $10 \mathrm{~Hz}$ and decreases to about $0.02 \mathrm{~s}$ at $50 \mathrm{~Hz}$. The result in Fig. 11 suggest that $\Delta \kappa_{0}(f)$, corresponding to the difference between $\kappa_{0}(f)$ at the surface and at depth, depends on frequency. The difference is highest at lower frequencies $\left(\Delta \kappa_{0}=0.06 \mathrm{~s}\right.$ at $\left.10 \mathrm{~Hz}\right)$ and decreases down to almost $0 \mathrm{~s}$ at $50 \mathrm{~Hz}$. This effect could be caused by true frequency-dependent site attenuation similar to the observations made by Cabas et al. (2017). The stronger difference in $\Delta \kappa_{0}$ could, however, also be caused by amplification (compare to Fig. 4) corresponding to a steeper slope that is only observed at the surface but not within the borehole.

We also computed a frequency-independent $\kappa_{0}$ using the kappa model with manually selected frequency bands. $\kappa_{0}=0.027 \pm 0.002 \mathrm{~s}$ and $\kappa_{0}=$ $0.064 \pm 0.002 \mathrm{~s}$ are obtained for station '06' and station '01', respectively. The results with the corresponding error ranges are shown in dashed in Fig. 11. The mean frequency range for the kappa model analysis spans from $12-40 \mathrm{~Hz}$ for station '06' and $10-28 \mathrm{~Hz}$ for station '01'. The $\kappa_{0}$ values of station '06' and '01' agree well with the results of the zeta model. The dashed line and bold curves intersect at around $27 \mathrm{~Hz}$ for station '06' and at around $19 \mathrm{~Hz}$ for station '01'. These values correspond to the centers of the averaged frequency bands chosen to derive $\kappa_{0}$ for station '06' and station '01'. That the $\kappa_{0}$ curves of the zeta and the kappa model intersect in the center of the frequency band follows naturally from the equivalence of $\kappa_{\eta, \zeta}$ and $\kappa$ for the same center frequency $f_{\text {cen }}$ as shown in Fig. 10. We want to point out again that $\kappa_{0}(f)$ for station ' 01 ' is reasonable and comparable to kappa model results even though the parameters $\zeta$ and $\eta$ are not physical.

\section{Comparison to other studies}

The $\kappa_{0}$ estimates and the errors from the studies of Ktenidou et al. (2015) and Papaioannou (2007) are shown for comparison in Fig. 11. Ktenidou et al. (2015) estimated $\kappa_{0}=0.021 \pm 0.008 \mathrm{~s}$ and $\kappa_{0}=0.061 \pm 0.011 \mathrm{~s}$ for station '06' and station '01', respectively. Papaioannou (2007) gives values of $\kappa_{0}=0.026 \mathrm{~s}$ and $\kappa_{0}=0.09 \mathrm{~s}$ for the two stations. Ktenidou et al. (2015) report that on average the range $15-35 \mathrm{~Hz}$ was used in their analysis. The error of the estimates and the utilized frequency range of Papaioannou (2007) are not reported. For station '06', the result of Papaioannou (2007) agrees well with the results of this study. The $\kappa_{0}$ estimate of Ktenidou et al. (2015) is lower than our results and does not intersect the curve obtained with 
the zeta model. However, our $\kappa_{0}$ results are still within the error range of Ktenidou et al. (2015). The agreement between our results and the one of Ktenidou et al. (2015) are better for station '01' while Papaioannou (2007) report a very large value of $\kappa_{0}$ for this station. Some differences between the results of this study and the other studies might not be related to differences in the method. They could also be attributed to differences in the datasets or the record and spectrum processing (Ji et al., 2020).

We compare our estimates of $\eta$ to several attenuation studies conducted in Greece that derived a frequency-dependent Q of the form given in Eq. 10. Hatzidimitriou (1993) derived coda Q using earthquake data from the Thessaloniki area in northern Greece with the single backscattering model of Aki and Chouet (1975). Coda Q is assumed to be equal to shear-wave Q as coda waves are mainly composed of scattered S-waves. They found that $\eta$ stabilizes around a value of 0.75 for different choices of the lapse time window. Hatzidimitriou (1995) report $\eta=0.91 \pm 0.07$ from the analysis of shear waves in northern Greece. Polatidis et al. (2003) derived $\eta=0.91$ from the decay of S-wave spectral amplitudes with distance in the back-arc Aegean Sea. Our estimated values of $\eta$ for the four deepest station of the EUROSEISTEST array $(\eta=0.7-0.84$, compare to Fig. 5 , right) lie within the range of the reported $\eta$ values for northern Greece.

The parameter $\eta$ of the Q studies listed above is derived from numerous events recorded at several stations and can be assumed to be region-specific. The results for the two topmost stations in Fig. 5 (right) suggest that $\eta$ derived with the zeta model is not only caused by the crustal waveguide but that the shallow layers could have their own value. The $\eta$ value would then be a net effect of the site and the region. If this is the case, $\eta$ as derived with the zeta model may not be directly comparable to the results of regional $\mathrm{Q}$ studies. We mentioned earlier, that we can also not guarantee that sensor '01' and '02' are unaffected by site resonance or downgoing waves. These effects can also contribute to the value of $\eta$ and generate the differences that we observe to the value of $\eta$ derived by other $\mathrm{Q}$ studies.

\section{Variability of $\eta$}

The exponent $\eta$ is linked to the curvature of the spectrum. If $\eta=0$, the decay is linear and the result is equivalent to the kappa model. The proper estimation of $\eta$ is the most critical step in the new approach. Fig. 5 shows that 
estimates of $\eta$ can be very different for different events. The single-recorded estimates of $\eta$ do not show any clear trend with distance, backazimuth or event magnitude (not shown here) that could explain the between-record differences.

We performed some synthetic tests using a modified version of the stochastic code of Boore (2003) where we introduced a frequency-dependent Q similar to Eq. 10. We observed, that with a single realization, it was often not possible to extract the correct input- $\eta$. Only the inversion of several stochastic spectra yielded reliable estimates of $\eta$. This is further confirmed by the observation that $\zeta$ shows only a trend with epicentral distance if $\eta$ is common for all recordings. Using the simulations, we noticed that $\eta$ is better resolved for stronger attenuation (low $Q_{0}$ ) and at larger distances where $\mathrm{Q}$ has more impact and where the spectral decay is stronger. At EUROSEISTEST, many recordings are from short distances (Fig. 2). We assume that the large scatter between individual observations of $\eta$ in Fig. 5 is mainly caused by the limited distance range.

Some of the record-to-record variability that we find for $\eta$ may be regarded as similar to the within-station variability that Ji et al. (2020) and Bora et al. (2017) show for $\kappa_{0}$. They report that the within-station variability of $\kappa_{0}$ can be even larger than the variability of $\kappa_{0}$ between different stations. Ji et al. (2020) attributed this effect to dataset choices, the record, and the spectrum processing. They observed, for example, that the estimated value of $\kappa$ is strongly orientation-dependent if only a single horizontal component is considered. The effect can be reduced by taking the average of two horizontal components, as we did in our analysis. They also argued that the focal depth, and therefore varying incidence angles, could contribute to the within-station scatter of $\kappa$. Bora et al. (2017) attribute the variability in $\kappa_{0}$ to depth variations of $\mathrm{Q}$. As seismic waves of different earthquakes reach the station from a range of distances, they may sample different depths and therefore different attenuation regimes. We can assume that these observations may be also true for the zeta model and that they partially explain the variability that we see for $\eta$. More research is needed to understand the within-station variability of $\eta$ and the driving factors that contribute to it. 


\section{Conclusion}

$\kappa$ has been applied in many studies for quantifying attenuation. As being a purely empirical parameter, frequency-dependence could not be ruled out. We, therefore, modified the kappa model of Anderson and Hough (1984) to account for the nonlinear spectral decay at high frequencies. This was done by incorporating a frequency-dependent $\mathrm{Q}$ that is modeled by a power-law function of the form $f^{\eta}$. In the new zeta model, two parameters $(\zeta$ and $\eta)$ are used to describe the nonlinear slope, in contrast to the original parameter $\kappa$. Similar to $\kappa, \zeta$ can be split into a path-dependent and a path-independent component to account for different effects between source and site.

We applied the new model, which we call the zeta $(\zeta)$ model, to borehole recordings of the EUROSEISTEST array in Greece. The zeta model delivers results that are consistent with the kappa model (Figs 10 and 11). Yet, the example in Fig. 8 shows that the zeta model is better able to capture the shape of the spectral decay in the sense that the standard deviation of the misfit reduces as well as the visual appearance of the model better matches the data, which is consistent with the suggested frequency dependence.

The intention of this publication is mainly to introduce the zeta model and to apply it to a first dataset. Future research could focus on, for example, the region-dependence of $\zeta$ and $\eta$ for different geological or tectonic regimes, the within-station variability of $\eta$ and the relationship to $\mathrm{Q}$ as measured by other methods. Moreover, the analyzed dataset is composed of weak

and moderate earthquake recordings. The kappa model of Anderson and Hough (1984), however, was developed for strong ground motion. One of the most important points is, therefore, to test the zeta model for strong ground motion.

Finally, it should be easy to incorporate the results of the zeta model in the computation of site amplification factors or ground-motion models. These models are already dependent on frequency and the new model slope is easily substituted.

\section{Data and Resources}

Data are taken from the EUROSEISTEST strong motion network as provided by: Research Unit of Soil Dynamics and Geotechnical Earthquake Engineering, Aristotle University of Thessaloniki (1993): EUROSEISTEST Strong 
Motion Network. International Federation of Digital Seismograph Networks. https://doi.org/10.7914/SN/EG (last accessed on February 12, 2020). A description of the database structure and content can be found in Pitilakis et al. (2013). Data analysis was partly performed using ObsPy, a Python framework for seismology (Beyreuther et al., 2010). Some plots were made using the Generic Mapping Tools version 5.4 .3 (www.soest.hawaii.edu/gmt; Wessel and Smith, 1998).

\section{Acknowledgements}

We are grateful to Fabian Bonilla and an anonymous reviewer for their constructive comments and questions that helped to improve the manuscript.

\section{References}

Aki, K. (1980a). Attenuation of shear-waves in the lithosphere for frequencies from 0.05 to $25 \mathrm{~Hz}$, Phys. Earth Planet. In. 21(1), 50-60.

Aki, K. (1980b). Scattering and attenuation of shear waves in the lithosphere, J. Geophys. Res. B Solid Earth Planets 85(B11), 6496-6504.

Aki, K., and B. Chouet (1975). Origin of coda waves: source, attenuation, and scattering effects, J. Geophys. Res. 80(23), 3322-3342.

Aki, K., and P. G. Richards (2002). Quantitative Seismology, University Science Books, Sausalito, California, US, 2nd edn.

Al Atik, L., A. Kottke, N. Abrahamson, and J. Hollenback (2014). Kappa $(\kappa)$ scaling of ground-motion prediction equations using an inverse random vibration theory approach, Bull. Seismol. Soc. Am. 104(1), 336-346.

Anderson, J. G., and S. E. Hough (1984). A model for the shape of the Fourier amplitude spectrum of acceleration at high frequencies, Bull. Seismol. Soc. Am. 74(5), 1969-1993.

Assimaki, D., W. Li, J. H. Steidl, and K. Tsuda (2008). Site amplification and attenuation via downhole array seismogram inversion: A comparative study of the 2003 Miyagi-Oki aftershock sequence, Bull. Seismol. Soc. Am. 98(1), 301-330. 
Beresnev, I. A. (2019). Interpretation of kappa and fmax filters as source effect, Bull. Seismol. Soc. Am. 109(2), 822-826.

Beyreuther, M., R. Barsch, L. Krischer, T. Megies, Y. Behr, and J. Wassermann (2010). ObsPy: A Python toolbox for seismology, Seismological Research Letters 81(3), 530-533.

Biro, Y., and P. Renault (2012). Importance and impact of host-to-target conversions for ground motion prediction equations in PSHA, in Proc. of the 15th World Conference on Earthquake Engineering, pp. 24-28, Lisbon, Portugal, September 24-28, 2012.

Bommer, J. J., K. J. Coppersmith, R. T. Coppersmith, K. L. Hanson, A. Mangongolo, J. Neveling, E. Rathje, A. Rodriguez-Marek, F. Scherbaum, R. Shelembe, P. Stafford, and F. Strasser (2013). A SSHAC Level 3 probabilistic seismic hazard analysis for a new-build nuclear site in South Africa, Earthquake Spectra 31(2), 661-698.

Boore, D. M. (2003). Simulation of ground motion using the stochastic method, Pure Appl. Geophys. 160, 635-676.

Bora, S. S., F. Cotton, F. Scherbaum, B. Edwards, and P. Traversa (2017). Stochastic source, path and site attenuation parameters and associated variabilities for shallow crustal European earthquakes, Bull. Earthquake Eng. 15(11), 4531-4561.

Brune, J. N. (1970). Tectonic stress and the spectra of seismic shear waves from earthquakes, J. Geophys. Res. 75, 4997-5009.

Cabas, A., A. Rodriguez-Marek, and L. F. Bonilla (2017). Estimation of sitespecific kappa $\left(\kappa_{0}\right)$-consistent damping values at KiK-Net sites to assess the discrepancy between laboratory-based damping models and observed attenuation (of seismic waves) in the field, Bull. Seismol. Soc. Am. 107(5), $2258-2271$.

Campbell, K. W. (2003). Prediction of strong ground motion using the hybrid empirical method and its use in the development of ground-motion (attenuation) relations in eastern North America, Bull. Seismol. Soc. Am. 93(3), 1012-1033. 
Carcolé, E., and H. Sato (2010). Spatial distribution of scattering loss and intrinsic absorption of short-period $S$ waves in the lithosphere of Japan on the basis of the Multiple Lapse Time Window Analysis of Hi-net data, Geophys. J. Int. 180(1), 268-290.

Edwards, B., O.-J. Ktenidou, F. Cotton, N. Abrahamson, C. Van Houtte, and D. Fäh (2015). Epistemic uncertainty and limitations of the $\kappa_{0}$ model for near-surface attenuation at hard rock sites, Geophys. J. Int. 202(3), $1627-1645$.

Efron, B., and C. Stein (1981). The jackknife estimate of variance, Ann. Math. Stat., pp. 586-596.

Eulenfeld, T., and U. Wegler (2016). Measurement of intrinsic and scattering attenuation of shear waves in two sedimentary basins and comparison to crystalline sites in Germany, Geophys. J. Int. 205(2), 744-757.

Fukushima, R., H. Nakahara, and T. Nishimura (2016). Estimating S-wave attenuation in sediments by deconvolution analysis of KiK-net borehole seismograms, Bull. Seismol. Soc. Am. 106(2), 552-559.

Galluzzo, D., M. La Rocca, L. Margerin, E. Del Pezzo, and R. Scarpa (2015). Attenuation and velocity structure from diffuse coda waves: Constraints from underground array data, Phys. Earth Planet. In. 240, 34-42.

Hanks, T. (1982). fmax, Bull. Seismol. Soc. Am. 72(6), 1867-1879.

Hatzidimitriou, P. M. (1993). Attenuation of coda waves in northern Greece, Pure Appl. Geophys. 140(1), 63-78.

Hatzidimitriou, P. M. (1995). S-wave attenuation in the crust in northern Greece, Bull. Seismol. Soc. Am. 85(5), 1381-1387.

Havskov, J., M. B. Sørensen, D. Vales, M. Özyazıcıŏ̆lu, G. Sánchez, and B. Li (2016). Coda Q in different tectonic areas, influence of processing parameters, Bull. Seismol. Soc. Am. 106(3), 956-970.

Hellweg, M., P. Spudich, J. Fletcher, and L. Baker (1995). Stability of coda Q in the region of Parkfield, California: View from the US Geological Survey Parkfield dense seismograph array, J. Geophys. Res. B Solid Earth Planets 100(B2), 2089-2102. 
Ji, C., A. Cabas, F. Cotton, M. Pilz, and D. Bindi (2020). Within-station variability in kappa: Evidence of directionality effects, Bulletin of the Seismological Society of America 110(3), 1247-1259.

Jongmans, D., K. Pitilakis, D. Demanet, D. Raptakis, J. Riepl, C. Horrent, G. Tsokas, K. Lontzetidis, and P.-Y. Bard (1998). EURO-SEISTEST: determination of the geological structure of the Volvi basin and validation of the basin response, Bull. Seismol. Soc. Am. 88(2), 473-487.

Knopoff, L. (1964). Empirical estimation of high-frequency ground motion on hard rock, Rev. Geophys. 2(4), 625-660.

Ktenidou, O.-J., C. Gélis, and L.-F. Bonilla (2013). A study on the variability of kappa $(\kappa)$ in a borehole: Implications of the computation process, Bull. Seismol. Soc. Am. 103(2A), 1048-1068.

Ktenidou, O.-J., F. Cotton, N. A. Abrahamson, and J. G. Anderson (2014). Taxonomy of $\kappa$ : A review of definitions and estimation approaches targeted to applications, Seismol. Res. Lett 85(1), 135-146.

Ktenidou, O.-J., N. A. Abrahamson, S. Drouet, and F. Cotton (2015). Understanding the physics of kappa $(\kappa)$ : Insights from a downhole array, Geophys. J. Int. 203(1), 678-691.

Mayor, J., S. S. Bora, and F. Cotton (2018). Capturing regional variations of hardrock $\kappa_{0}$ from coda analysis, Bull. Seismol. Soc. Am. 108(1), 399-408.

Papageorgiou, A. S. and K. Aki (1983). A specific barrier model for the quantitative description of inhomogeneous faulting and the prediction of strong ground motion. I. Description of the model, Bull. Seismol. Soc. Am. 73(3), 693-722.

Papaioannou, C. (2007). A preliminary study for the distance dependence of the spectral decay parameter $\kappa$ for the Euroseisrisk strong motion array, Proceedings of the 4ICEGE, pp. 25-28.

Parolai, S., and D. Bindi (2004). Influence of soil-layer properties on k evaluation, Bull. Seismol. Soc. Am. 94(1), 349-356.

Pasyanos, M. E. (2013). A lithospheric attenuation model of North America, Bull. Seismol. Soc. Am. 103(6), 3321-3333. 
Perron, V., A. Laurendeau, F. Hollender, P.-Y. Bard, C. Gélis, P. Traversa, and S. Drouet (2018). Selecting time windows of seismic phases and noise for engineering seismology applications: A versatile methodology and algorithm, Bull. Earthquake Eng. 16(6), 2211-2225.

Pitilakis, K., D. Raptakis, K. Lontzetidis, T. Tika-Vassilikou, and D. Jongmans (1999). Geotechnical and geophysical description of Euro-Seistest, using field, laboratory tests and moderate strong motion recordings, $J$. Earthq. Eng. 3(3), 381-409.

Pitilakis, K., Z. Roumelioti, D. Raptakis, M. Manakou, K. Liakakis, A. Anastasiadis, and D. Pitilakis (2013). The EUROSEISTEST strong-motion database and web portal, Seismol. Res. Lett. 84(5), 796-804.

Polatidis, A., A. Kiratzi, P. Hatzidimitriou, and B. Margaris (2003). Attenuation of shear-waves in the back-arc region of the Hellenic arc for frequencies from 0.6 to $16 \mathrm{~Hz}$, Tectonophysics 367(1-2), 29-40.

Purvance, M. D., and J. G. Anderson (2003). A comprehensive study of the observed spectral decay in strong-motion accelerations recorded in Guerrero, Mexico, Bull. Seismol. Soc. Am. 93(2), 600-611.

Raptakis, D., M. Manakou, F. Chávez-García, K. Makra, and K. Pitilakis (2005). 3D configuration of Mygdonian basin and preliminary estimate of its site response, Soil Dyn. Earthq. Eng. 25(11), 871-887.

Sato, H., M. C. Fehler, and T. Maeda (2012). Seismic wave propagation and scattering in the heterogeneous earth, vol. 484, Springer.

Shi, J., W.-Y. Kim, and P. G. Richards (1996). Variability of crustal attenuation in the northeastern United States from Lg waves, J. Geophys. Res. $B$ Solid Earth Planets 101(B11), 25231-25242.

Singh, S., and R. B. Herrmann (1983). Regionalization of crustal coda Q in the continental United States, J. Geophys. Res. B Solid Earth Planets 88(B1), 527-538.

Toksöz, M. N., A. M. Dainty, E. Reiter, and R.-S. Wu (1988). A model for attenuation and scattering in the Earth's crust, pp. 81-100, Birkhäuser Basel, Basel. 
Van Houtte, C., S. Drouet, and F. Cotton (2011). Analysis of the origins of $\kappa$ (Kappa) to compute hard rock to rock adjustment factors for GMPEs, Bull. Seismol. Soc. Am. 101(6), 2926-2941.

Wang, W. and P. Shearer (2019). An improved method to determine codaQ, earthquake magnitude, and site amplification: Theory and application to southern California, J. Geophys. Res. B Solid Earth Planets 124(1), $578-598$.

Wessel, P., and W. H. F. Smith (1998). New, improved version of generic mapping tools released, EOS, Trans. Am. Geophys. Un. 79(47), 579. 
Helmholtz Centre Potsdam

German Research Centre for Geosciences GFZ

Telegrafenberg

14473 Potsdam, Germany

ahaendel@gfz-potsdam.de

(A. Haendel, M. Pilz, F. Cotton)

Seismological Laboratory

University of Nevada

1664 N. Virginia Street

Reno

Nevada 89557, US

(J.G. Anderson) 